\title{
Foundations for a Graph-Based Approach to the Specification of Access Control Policies ${ }^{\star}$
}

\author{
Manuel Koch ${ }^{1}$, Luigi Vincenzo Mancini ${ }^{2}$, and Francesco Parisi-Presicce ${ }^{2,3}$ \\ 1 PSI AG, Berlin (DE) \\ 2 Univ. di Roma La Sapienza, Rome (IT) \\ 3 George Mason Univ., Fairfax VA (USA) \\ mkoch@psi.de lv.mancini@dsi.uniroma1.it parisi@dsi.uniroma1.it; \\ fparisi@ise.gmu.edu
}

\begin{abstract}
Graph Transformations provide a uniform and precise framework for the specification of access control policies allowing the detailed comparison of different policy models and the precise description of the evolution of a policy. Furthermore, the framework is used for an accurate analysis of the interaction between policies and of the behavior of their integration with respect to the problem of conflicting rules. The integration of policies is illustrated using the Discretionary Access Control and the Lattice Based Access Control policies.
\end{abstract}

\section{Introduction}

A considerable amount of work has been carried out recently on models and languages for Access Control. Access Control (AC) is concerned with determining the activities of legitimate users [SS94]. Usually AC is enforced by a reference monitor which mediates every attempted access by a subject (a program executing on behalf of a user) to objects in the system. In KMPP00b KMPP00a we have proposed graph transformations as a uniform conceptual framework for the specification of access control policies. In this paper we discuss the formal properties of this framework and their applications to problems not addressed anywhere else in a formal way.

The three main AC policies commonly used in computer systems are discretionary policies [SS94], lattice-based policies (also called mandatory policies) [San93] and role-based policies [San98]. As illustrative examples, we use here the lattice-based access control (LBAC) and the access control list (ACL) that is an implementation of a discretionary policy. Role-based access control is not considered in this article, but is the main focus of [KMPP00b].

Lattice-based access control: Classic LBAC enforces unidirectional information flow in a lattice of security levels 1 . The diagram on the left-hand side of Fig. [1 shows a partial order security lattice.

\footnotetext{
* partially supported by the EC under TMR Network GETGRATS and under Esprit WG APPLIGRAPH, and by the Italian MURST.

${ }^{1}$ In San93, security levels are called security labels. We use 'security level' here to avoid confusion with the notion of a label for a node or an edge in a graph.
} 


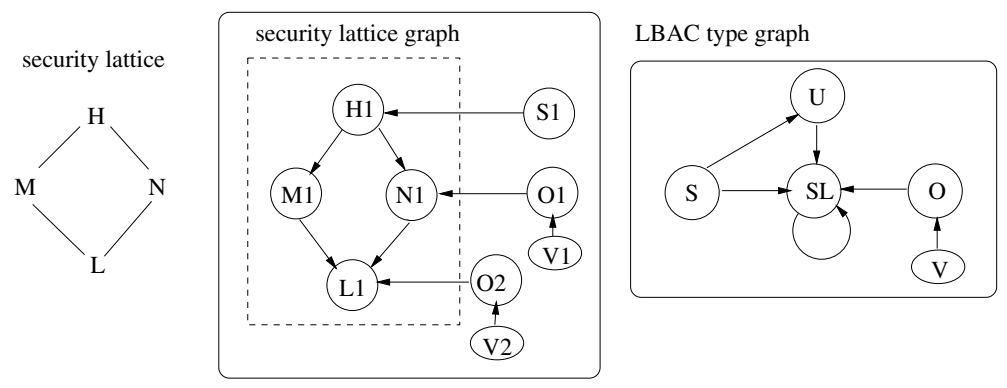

Fig. 1. A security lattice (left-hand side) and its presentation by a graph (middle). The type graph for the LBAC model (right-hand side).

The LBAC policy is expressed in terms of security levels attached to subjects and objects. A subject is a process in the system and each subject is associated to a single user, where one user may have several subjects concurrently running in the system. An object is a container of information, e.g. files or directories in an operating system. Usually the security levels on subjects and objects, once assigned, do not change. If $\lambda(x)$ denotes the security level of $x$ (subject or object) then the specific LBAC rules for a lattice allow a subject $S$ to read object $O$ if $\lambda(S) \geq \lambda(O)$ and to write object $O$ if $\lambda(S)=\lambda(O)$.

Access Control List: The ACL policy is an implementation of a discretionary AC policy. We consider an ACL policy similar, but simpler, to that one used in the UNIX operating system. Our model distinguishes only between the owner of an object and the rest of the world and for simplicity groups are not considered. The owner of the object has read, write and execution access and can change the access permissions of the object with respect to the world.

The different models for Access Control have been specified with expressive but ad hoc languages requiring ad hoc conversions to compare the relative strengths and weaknesses. Not much work BdVS00] has been performed on the evolution of a policy, to construct in an incremental way complex policies, and on the integration of policies, to obtain the global policy of an organization by combining the security policies of different departments.

The main goal of this paper is to present some basic properties of a formal model for Access Control policies based on graphs and graph transformations and to address the problems of evolution and integration in a categorical setting. A system state is represented by a graph and graph transformation rules describe how a system state evolves. The specification ("framework") of an AC policy contains also declarative information ("invariants") on what a system graph must contain (positive) and what it cannot contain (negative). A crucial property of a framework is that it specifies a coherent policy (one without internal contradictions). As with any soft system, policies can evolve and can be combined: we have formalized here in a categorical setting the notions of evolution and of integration and their main properties. To help with the issue of conflicting rules in the integrated framework obtained by combining two distinct ones, the notion of metapolicy is proposed. 
The paper is organized as follows: the next section reviews graph transformations and introduces the LBAC and the ACL policies; Sect. 3 defines the formal framework to specify AC policies and presents its main properties; Sect. 4 discusses the notion of integration of policies and Sect. 5 introduces metapolicies to resolve conflicts; the last section mentions related and future work.

\section{Graph Transformations}

This section introduces graph transformations [Roz97. The LBAC model is used throughout the section to support the explanations by an example.

A graph $G=\left(G_{V}, G_{E}, s_{G}, t_{G}, l_{G}\right)$ consists of disjoint sets of nodes $G_{V}$ and edges $G_{E}$, two total functions $s_{G}, t_{G}: G_{E} \rightarrow G_{V}$ mapping each edge to its source and target node, respectively, and a function $l_{G}: G_{V} \cup G_{E} \rightarrow L$ assigning to each node/edge a label. Labels are elements of a set $L=X \cup C$, where $X$ is a set of variables and $C$ is a set of constants. A binary relation $\prec \subseteq L \times L$ is defined on $L$ as $\alpha \prec \beta$ if and only if $\alpha \in X$. A path between nodes $a$ and $b$ is indicated by an edge $a \stackrel{*}{\rightarrow} b$ and can be seen as an abbreviation for a set of paths each representing a possible sequence of edges between $a$ and $b$.

A total graph morphism $f: G \rightarrow H$ between graphs $G=\left(G_{V}, G_{E}, s_{G}, t_{G}, l_{G}\right)$ and $H=\left(H_{V}, H_{E}, s_{H}, t_{H}, l_{H}\right)$ is a pair $\left(f_{V}, f_{E}\right)$ of total mappings $f_{V}: G_{V} \rightarrow$ $H_{V}$ and $f_{E}: G_{E} \rightarrow H_{E}$ that respect the graph structure, i.e. $f_{V} \circ s_{G}=s_{H} \circ f_{E}$ and $f_{V} \circ t_{G}=t_{H} \circ f_{E}$, as well as the label order, i.e. $l_{G}(v) \prec l_{H}\left(f_{V}(v)\right)$ for each $v \in G_{V}$ and $l_{G}(e) \prec l_{H}\left(f_{E}(e)\right)$ for each $e \in G_{E}$. A partial graph morphism $f$ : $G \rightarrow H$ is a total graph morphism $\bar{f}: \operatorname{dom}(f) \rightarrow H$ from a subgraph $\operatorname{dom}(f) \subseteq$ $G$ to $H$. Graphs and partial graph morphisms form a category $\mathbf{G r a p h}^{\mathbf{P}}$. The subcategory of graphs and total graph morphisms is denoted by Graph. The category $\mathbf{G r a p h}^{\mathbf{P}}$ is in general not co-complete, but has pushouts for morphisms $f_{p}: G \rightarrow H$ and $f_{c}: G \rightarrow K$ where $f_{p}$ is label preserving, i.e. $l_{G}(x)=l_{H}\left(f_{p}(x)\right)$ for all $x \in G$ (node or edge) PPEM87.

A type graph $T G$ represents the type information in a graph transformation system [CELP96, specifying the node and edge types which may occur in the instance graphs modeling system states. For instance, the type graph in Fig. 1] on the right shows the possible types for the LBAC graph model. There is a type $U$ for user nodes, a type $S$ for subjects, a type $O$ for objects, a type $V$ for the value of objects and a type $S L$ for the security levels. This type graph indicates also that there cannot be an edge from a node of type $U$ to a node of type $O$.

A pair $\left\langle G, t_{G}\right\rangle$, where $G$ is a graph and $t_{G}: G \rightarrow T G$ is a total graph morphism, is called a graph typed over $T G$. If the type graph is fixed, we denote the pair simply as $G$. The total graph morphism $t_{G}$ is called typing morphism and is indicated in the examples by the symbols used for nodes and edges. In the middle of Fig. 1 a graph typed over the LBAC type graph is shown. The graph consists of a node with label $H 1$ and type $S L$, a node with label $S 1$ and type $S$, a node with label $O 1$ and type $O$, etc. The security lattice on the left-hand side is modeled by a security lattice graph. From now on, the typing morphism maps a node with label $T x$ to the type $T$. 
A morphism between typed graphs $\left\langle G, t_{G}\right\rangle$ and $\left\langle H, t_{H}\right\rangle$ is given by a partial graph morphism $f: G \hookleftarrow \operatorname{dom}(f) \rightarrow H$ that preserves types, that is, the diagram

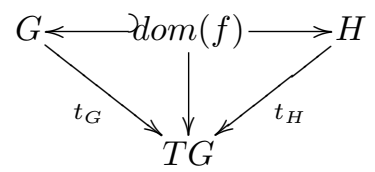

in Graph commutes. The morphism is total if the underlying graph morphism is total. Graphs typed over a fixed type graph $T G$ and morphisms between them form a category TG [CELP96]. The existence of pushouts is inherited from the category Graph ${ }^{\mathbf{P}}$.

A graph typed over a type graph $T G$ can be re-typed over $T G^{\prime}$ if there is a total morphism $f: T G \rightarrow T G^{\prime}$. The re-typing of a graph $\left\langle G, t_{G}\right\rangle$ typed over $T G$ to a graph typed over $T G^{\prime}$ is a renaming of types in $G$. Re-typing from $T G^{\prime}$ to $T G$ is a renaming of types and a forgetting of nodes and edges. Formally, the re-typing w.r.t. a morphism $f: T G \rightarrow T G^{\prime}$ is specified by functors $F_{f}: \mathbf{T G} \rightarrow \mathbf{T G}^{\prime}$ and $V_{f}: \mathbf{T G}^{\prime} \rightarrow \mathbf{T G}$, called forward typing and backward typing functor CELP96GRPPS98.

A graph rule $p: r$, or just rule, is given by a rule name $p$, from a set $R$ Names, and a label preserving morphism $r: L \rightarrow R$. The graph $L$, left-hand side, describes the elements a graph must contain for $p$ to be applicable. The partial morphism is undefined on nodes/edges that are intended to be deleted, defined on nodes/edges that are intended to be preserved. Nodes and edges of $R$, righthand side, without a pre-image are newly created.

Example 1 (Graph rules for the LBAC graph model). Figure 2 shows the rules for the LBAC policy. The labels for the nodes $(U x, S x, S L x, S L y, \ldots)$ of the rules are variables taken from the set of variables in $L$. The rule new object creates a new object $O x$ connected to a node $V x$ (the initial value of the object). The object
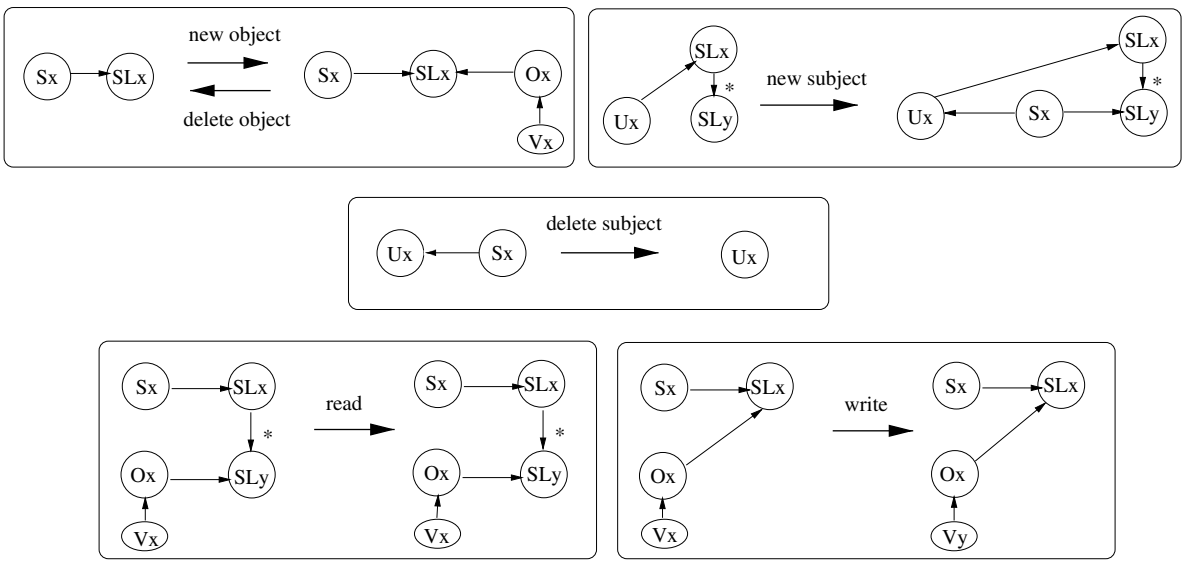

Fig. 2. Graph rules for the LBAC policy. 
$O x$ gets the security level $S L x$. The variable $S L x$ is generic: it is substituted by the actual security level of the subject when the rule is applied. The rule for delete object for the deletion of objects is represented by reversing the partial morphism of the rule new object. The rule new subject creates a subject $S x$ on behalf of a user $U x$. The new subject is attached to a security level $S L y$ that is lower in the hierarchy graph than the security level $S L x$ of the user $U x$. This requirement is specified by the path from $S L x$ to $S L y$, since edges in the security lattice graph point from higher to lower levels.

For the application of rules we use the Single Pushout (SPO) approach to graph transformations $\mathrm{EHK}^{+} 97$. Formally, the application of a graph rule $p$ : $L \stackrel{r}{\rightarrow} R$ to a graph $G$ is given by a total graph morphism $m: L \rightarrow G$, called match for $p$ in $G$. The direct derivation $G \stackrel{p, m}{\Rightarrow} H$ from $G$ to the derived graph $H$ is given by the pushout of $r$ and $m$ in TG (see the diagram below). Note that the pushout exists, since the rule morphism is label preserving [PPEM87].

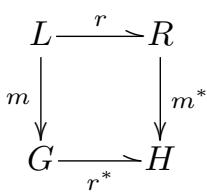

Example 2 (Application of a graph rule). In Fig. 3, the left-hand side $L$ of the rule new subject occurs several times in $G$. In one possible match the node $U x$ in $L$ is associated to the node $U_{2}$ in $G$ and the nodes $S L x$ and $S L y$ to the specific security level $H$.

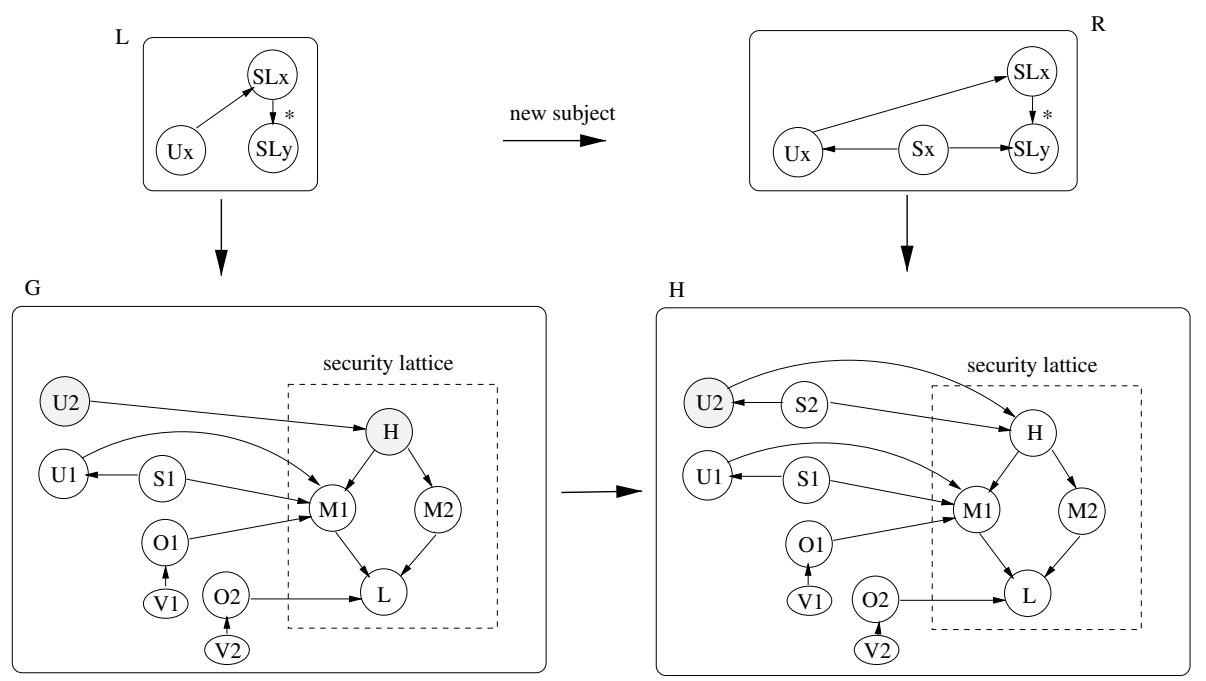

Fig. 3. Application of rule new subject. 
For the specification of the ACL by graph transformations, negative application conditions for rules are needed. A negative application condition (NAC) for a rule $p: L \stackrel{r}{\rightarrow} R$ consists of a set $A(p)$ of pairs $(L, X)$, where the graph $L$ is a subgraph of $X$. The part $X \backslash L$ represents a structure that must not occur in a graph $G$ for the rule to be applicable. In the figures, we depict $(L, X)$ by the graph $X$, where the subgraph $L$ is drawn with solid and $X \backslash L$ with dashed lines. A rule $p: L \stackrel{r}{\rightarrow} R$ with a $\operatorname{NAC}(L, X)$ is applicable to $G$ if $L$ occurs in $G$ and it is not possible to extend $L$ to $X$. Examples of rules with a NAC are the ACL rules connect and give read in Fig. 5 .

Example 3 (Graph rules for the $A C L$ ). The type graph $T G_{A C L}$ in Fig. 4 provides the node types $U, O$ and $P$. Just as in the LBAC model, a node of type $U$ represents a user and a node of type $O$ an object. An edge between a user node $U$ and an object node $O$ specifies that $U$ is the owner of the object $O$. A node

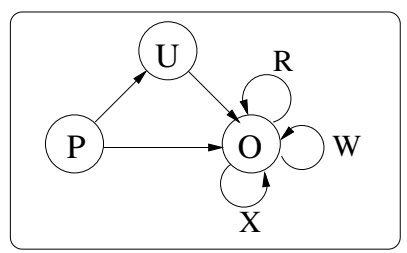

Fig. 4. The type graph for the ACL.

of type $P$ represents a process. An edge of type $R, W$ or $X$ represents the read, write or execute permission of an object to the world. The owner of the object has always all the permissions for his/her objects and does not need the loops. Some of the ACL graph rules are shown in Fig. 15. The rule new process starts a new process on behalf of a user. To kill a process, the rule remove process deletes the process node and its connection to the user. The rule create object adds a new node $O x$ to the system, connecting it to the process node $P x$ that
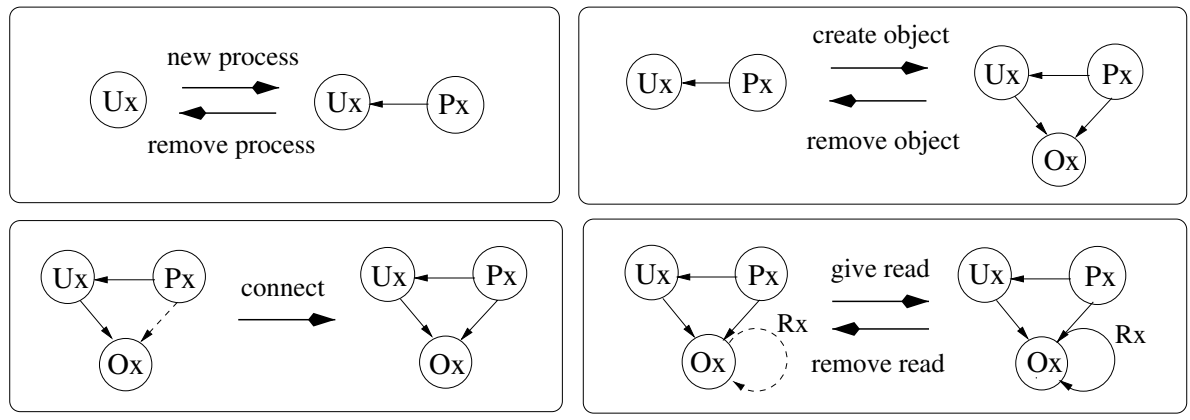

Fig. 5. Graph rules for the ACL model. 
has created the object and to the user node $U x$ to which the process belongs. The rule connect connects a process of a user to an object of the user. The rule has a NAC (indicated by the dashed edge between $P x$ and $O x$ on the left-hand side of the rule) that forbids the application of the rule to processes and objects of the user already connected. The rule give read gives the read permission provided that it has not been granted already. Other rules such as give write and give execution are not shown.

\section{$3 \quad$ Security Policy Framework}

This section introduces the framework for the specification of AC policies based on graph transformations. The framework is called security policy framework and consists of four components: The first component is a type graph that provides the type information of the AC policy. The second component is a set of graph rules specifying the policy rules that generate the graphs representing the states of the system accepted by the AC policy. For some AC policies, it is meaningful to restrict the set of system graphs constructed by the graph rules, since not all of them represent valid states. Therefore, a security policy framework contains also two sets of constraints that specify graphs that shall not be contained in any system graph (negative constraints) and graphs that must be explicitly constructed as parts of a system graph (positive constraints). In the actual implementation of an AC policy, the constraints are redundant since the only acceptable states are those explicitly built by the implemented rules. But when developing an AC policy through successive refinement steps, or when comparing different policies, or when trying to predict the behavior of the policy obtained by integrating two different ones, it is useful to have the additional information provided by the constraints. Furthermore, it is usually difficult to extract negative informations from "constructive" rules. Positive and negative constraints can be considered as formal documentation of the initial requirements and the development process of rules. Both positive and negative constraints are formally specified by morphisms. Only their semantics distinguishes them.

Definition 1 (Negative and positive constraints). $A$ constraint (positive or negative) is given by a total graph morphism $c: X \rightarrow Y$. A graph $G$ satisfies a positive (negative) constraint $c$ if for each total graph morphism $p: X \rightarrow G$ there exists (does not exist) a total graph morphism $q: Y \rightarrow G$ such that $X \stackrel{c}{\rightarrow}$ $Y \stackrel{q}{\rightarrow} G=X \stackrel{p}{\rightarrow} G$.

Example 4 (Constraints for $L B A C$ and $A C L$ ). Figure 6 shows two positive constraints and two negative constraints for the LBAC model. The morphisms for the negative constraints are the identity on the graphs shown. The positive and the negative constraint on the left-hand side require that objects always have a security level (the positive constraint) and that there does not exist more than one security level for an object (negative constraint). The right-hand side of the figure specifies the same existence and uniqueness requirements for subjects.

The constraints for the ACL framework in Fig. 7require that a process belong to a unique user (the positive and the first negative constraint), that an object 
positive constraints :
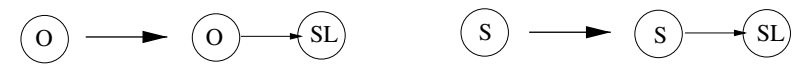

negative constraints :
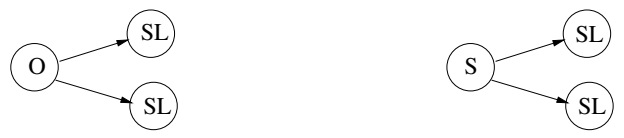

Fig. 6. Positive and negative constraints for LBAC.

does not belong to more than one user (the second negative one) and there is at most one permission loop with the same permission attached to the same object (the third negative one). Note that the third negative constraint represents three negative constraints, one for $R$, one for $W$ and one for $X$.

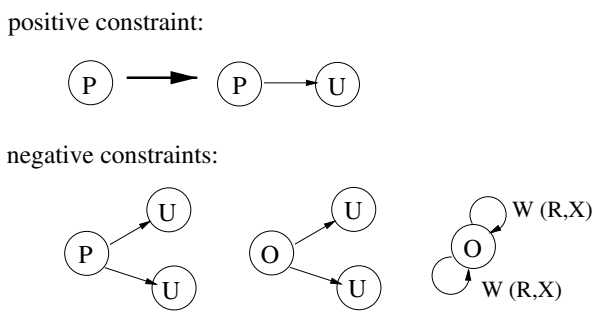

Fig. 7. Positive and negative constraints for the ACL.

Definition 2 (Security Policy Framework). A security policy framework, or just framework, is a tuple $S P=\left(T G,\left(P, r_{P}\right)\right.$, Pos, Neg), where $T G$ is a type graph, the pair $\left(P, r_{P}\right)$ consists of a set of rule names and a total mapping $r_{P}: P \rightarrow|\operatorname{Rule}(T G)|$ mapping each rule name to a rule $L \stackrel{r}{\rightarrow} R$ of $T G$-typed graphs, Pos is a set of positive and Neg is a set of negative constraints.

The graphs that can be constructed by the rules of a framework represent the system states possible within the policy model. These graphs are called system graphs in the sequel.

A security policy framework is positive (resp. negative) coherent if all system graphs satisfy the constraints in Pos (resp. Neg). It is coherent if it is both positive and negative coherent.

The security policy framework for the LBAC policy consists of the type graph in Fig. 1 and the negative and positive constraints in Fig. 6. The rule names $p_{1}, \ldots, p_{6}$ are mapped to the rules in Example 1 .

A security policy framework morphism $f: S P_{1} \rightarrow S P_{2}$, or just framework morphism, relates security policy frameworks by a total graph morphism $f_{T G}$ : 
$T G_{1} \rightarrow T G_{2}$ between the type graphs and a mapping $f_{P}: P_{1} \rightarrow P_{2}$ between the sets of rule names. The mapping $f_{P}$ must preserve the behavior of rules as in the sense that a rule name $x$ can be mapped to a rule name $f_{P}(x)$ only if $f_{P}(x)$ does on the renamed types everything which $x$ does and possibly more. The set of positive constraints in $S P_{2}$ can contain, in addition to $P_{0} s_{1}$, new positive constraints and positive constraints of $S P_{1}$ extended w.r.t. new types. The set of negative constraints in $S P_{2}$ may contain additional negative constraints on new types, but must not impose new negative constraints on old types.

Definition 3 (Framework Morphism). A framework morphism between security policy frameworks $S P_{i}=\left(T G_{i},\left(P_{i}, r_{P_{i}}\right), P_{o s}, N e g_{i}\right)$ for $i=1,2$ is a pair $f=\left(f_{T G}, f_{P}\right): S P_{1} \rightarrow S P_{2}$, where $f_{T G}: T G_{1} \rightarrow T G_{2}$ is a total graph morphism and $f_{P}: P_{1} \rightarrow P_{2}$ is a total mapping, so that $V_{f_{T G}}\left(r_{P_{2}}\left(f_{P}(p)\right)\right)=r_{P_{1}}(p)$ for all $p \in P_{1}$, Pos $_{1} \subseteq V_{f_{T G}}\left(\right.$ Pos $\left._{2}\right)$ and $V_{f_{T G}}\left(N e g_{2}\right) \subseteq N e g_{1}$.

We provide now the categorical formalization by defining the category of security policy frameworks and framework morphisms.

Definition 4 (Category of Security Policy Frameworks). The category of security policy frameworks, denoted by $\mathbf{S P}$, has as objects all security policy frameworks and as morphisms all framework morphisms. For each framework $S P, i d_{S P}=\left(i d_{T G}, i d_{P}\right)$ is the identity and composition is defined componentwise.

Proposition 1 (Initial Security Policy Framework). The initial object in SP is given by the security policy framework $S P_{I}=(\emptyset,(\emptyset, r), \emptyset, \emptyset)$.

Security policy frameworks can be glued together using the standard categorical constructions.

Proposition 2 (Pushouts). The category SP has all pushouts.

By combining the previous two, we obtain the main result of this section.

Theorem 1 (Colimits). The category $\mathbf{S P}$ is finitely cocomplete.

A security policy framework $S P=\left(T G,\left(P, r_{P}\right), P o s, N e g\right)$ can be changed by modifying its components, that is, the extension or reduction of the type graph, the addition/removal of a graph rule to/from $P$, the addition/removal of a positive constraint to/from Pos and the addition/removal of a negative constraint to/from $\mathrm{Neg}$. A framework morphism $f: S P_{1} \rightarrow S P_{2}$ describes the change of the framework $S P_{1}$ to the framework $S P_{2}$, but also from $S P_{2}$ to $S P_{1}$. We define an evolution as a sequence of framework morphisms in the category SP that can be travelled in both directions.

Definition 5 (evolution). An evolution of a framework $S P$ to a framework $S P^{\prime}$ is a sequence $e=\left(S P_{0} S P_{1} \ldots S P_{n-1} S P_{n}\right)$ of frameworks such that $S P_{0}=$ $S P, S P_{n}=S P^{\prime}$ and, for each $i=0, \ldots, n-1$, there is a framework morphism $m_{i}^{f}: S P_{i} \rightarrow S P_{i+1}$ or $m_{i}^{b}: S P_{i+1} \rightarrow S P_{i}$. 
The evolution of a security policy framework yields a new security policy framework that reflects the desired changes. The changes, however, do not ensure generally that the new security policy framework is coherent. From a semantical point of view, this problem can be solved by considering the full sub-category $\mathbf{S P}^{c}$ of $\mathbf{S P}$ that contains only coherent security policy frameworks. Evolution is possible only in this sub-category. From an operational point of view, we can solve the problem by using a mechanical construction originally introduced in HW95. The construction manipulates the rules of a framework by adding application conditions to ensure that the rules do not create graphs that do not satisfy the constraints. A methodology for generating a coherent security policy framework is presented in KMPP00b KMPP00a].

\section{Integration of Security Policy Frameworks by Pushouts}

Integration is concerned with the merging of AC policies. A merge is necessary on the syntactical level, i.e. a merge of the security policy frameworks, and on the semantical level, i.e. the merge of the system graphs representing the state at merge time. The merge on the semantical level is what distinguishes an evolution from an integration: evolution is expressed syntactically by considering only the security policy frameworks, integration includes semantical changes, too. The integration of two AC policies on the syntactical level is a pushout of the security policy frameworks in the category $\mathbf{S P}^{2}$. Two security policy frameworks $S P_{1}$ and $S P_{2}$ are related by an auxiliary framework $S P_{0}$ that identifies the common parts (types and rules) in both frameworks; the actual integration is expressed by framework morphisms $f_{1}: S P_{0} \rightarrow S P_{1}$ and $f_{2}: S P_{0} \rightarrow S P_{2}$. The pushout of $f_{1}$ and $f_{2}$ in $\mathbf{S P}$ integrates the frameworks $S P_{1}$ and $S P_{2}$ in a new security policy framework $S P$ called the integrated framework.

Throughout this section, the integration of the LBAC framework with the ACL framework (both introduced in Section 31) is used as an example.

Example 5 (Pushout integration of the ACL and LBAC frameworks). The type graph in the middle of Fig. 8 shows the types common to $A C L$ and $L B A C$. The $U$ and the $O$ types are in common and the type $P$ (processes in ACL) and the type $S$ (subjects in LBAC) coincide. The edge between the $U$ and the $P$ (resp. $S$ ) node is a common part as well. The pushout of the two type graphs is the type graph at the bottom of Fig. 8 . The pushout identifies the $P$ and $S$ node to a common type $P$. All rules are kept in the integrated security policy framework, where their graphs are now typed over the integrated type graph. The integrated policy framework contains the two positive constraints of the LBAC model (now typed over the integrated type graph) and the positive ACL constraint. The integrated framework has no negative constraints, since there are (after re-typing) no common negative constraints in $A C L$ and $L B A C$.

An important integration aspect is the preservation of coherence: if the frameworks $S P_{1}$ and $S P_{2}$ are coherent, is $S P$ ? Generally, this is not the case. The

\footnotetext{
2 The integration concepts of the paper can be easily generalized to an integration of several frameworks because of the existence of (finite) colimits in SP.
} 


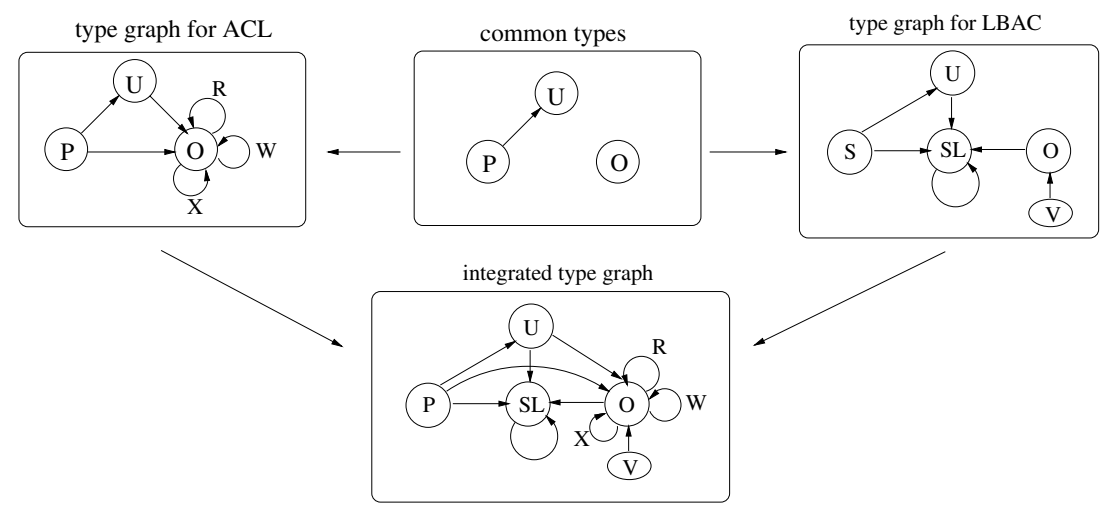

Fig. 8. Integrated type graph for the combined LBAC and ACL security model.

integrated framework for the ACL and the LBAC frameworks of the previous example contains a positive constraint that requires a security level for each node of type $P$ (stemming from the identification of the LBAC type $S$ and the ACL type $P$ ). This requirement can be destroyed by the ACL rule new process. Negative constraints, however, are preserved by the pushout.

Proposition 3 (Preservation of negative coherence). Given framework morphisms $f_{1}: S P_{0} \rightarrow S P_{1}$ and $f_{2}: S P_{0} \rightarrow S P_{2}$ so that $S P_{1}$ and $S P_{2}$ are coherent, the pushout object $S P=\left(T G^{\text {int }},\left(P^{\text {int }}, r_{P^{i n t}}\right)\right.$, Pos $\left.^{\text {int }}, N e g^{\text {int }}\right)$ of $f_{1}$ and $f_{2}$ in $\mathbf{S P}$ is coherent w.r.t. the set of negative constraints Negint.

Coherence w.r.t. positive constraints is generally not preserved by the pushout construction as the counterexample above shows. The reason for incoherence w.r.t. positive constraints, however, can be reduced to the parts of positive constraints referring to common types. Coherence of positive constraints referring to types occuring only in $S P_{1}$ or only in $S P_{2}$ is preserved.

Proposition 4 (Preservation of positive coherence). Given framework morphisms $f_{1}: S P_{0} \rightarrow S P_{1}$ and $f_{2}: S P_{0} \rightarrow S P_{2}$ with $S P_{1}$ and $S P_{2}$ coherent, the pushout $S P=\left(T G^{i n t},\left(P^{i n t}, r_{P^{i n t}}\right), P o s^{i n t}, N e g^{i n t}\right)$ of $f_{1}$ and $f_{2}$ in $\mathbf{S P}$ is incoherent if and only if $S P$ is incoherent w.r.t. positive constraints of Pos ${ }^{\text {int }}$ containing types in $T G_{0}$.

After merging the AC policies on the syntactical level to an integrated $S P$, the AC policies are merged on the semantical level by a pushout of the system graphs $G_{1}$ of $S P_{1}$ and $G_{2}$ of $S P_{2}$ representing the system states at merge time. The merge of the system graphs must yield a graph typed over the integrated type graph $T G^{\text {int }}$ of $S P$.

Example 6 (Integration of $L B A C$ and $A C L$ System Graphs). Figure 9 shows a system graph for the ACL framework and the LBAC framework. The auxiliary graph $G_{0}$ contains the user $U 1$ (may be working for both companies) and the objects $O 1$ and $O 2$ (may be files already shared before the merge). The integrated system graph at the bottom of Fig. 9] contains features of both frameworks. 
system graph for the ACL model

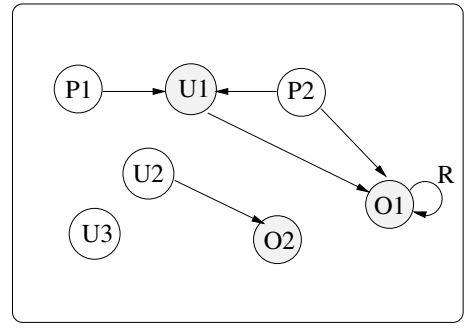

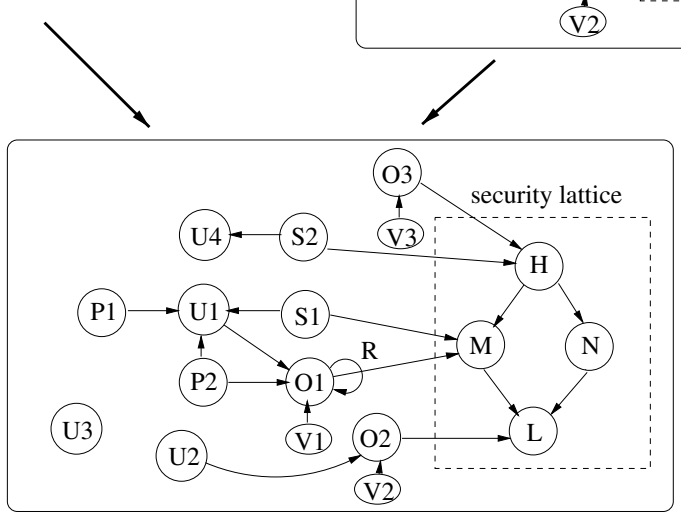

system graph for the LBAC model

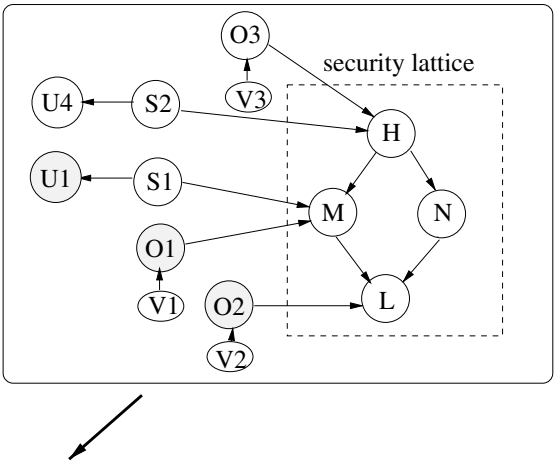

Fig. 9. Integrated system graph for the combined LBAC and ACL framework.

The integration of the system graphs does not ensure that the integrated system graph satisfies the constraints of the integrated framework $S P$. There are two possible solutions: first, the integrated system graph is adapted to satisfy the constraints. In the example, the integrated system graph in Fig. 9] could be modified by connecting objects and processes without security level with the security lattice graph. Second, the set of constraints can be changed by, for instance, removing the unsatisfied constraints. This is meaningful, if one policy is preferred and the constraint is from the other policy. In the example, the LBAC constraint for security levels could be removed if the ACL policy is preferred.

\section{Solving Rule Conflicts by Meta Policies}

In this section, we assume that two security policies are integrated by a pushout in $\mathbf{S P}$ for the frameworks and a pushout in $\mathbf{T} \mathbf{G}^{i n t}$ for the system graphs. Even if the integrated system graph is modified to be coherent w.r.t. the constraints of the integrated framework, there may be rules in the integrated framework, coming from $S P_{1}$ and $S P_{2}$, respectively, that are applicable to the same part of the integrated system graph. If the rules specify two conflicting actions specific to the policy, we call such a situation a rule conflict and denote by $C R(S P)$ the set of conflicting rule pairs. For instance, the LBAC rule new object and the ACL rule create object are in conflict, since both are applicable to the user 
$U 1$ in the integrated system graph in Fig. 9. The rule new object would create an object with a security level, the rule create object an object without one. Which rule shall be applied in this case? Several strategies, called meta policies, are possible to resolve conflicts between rules. A meta policy is specified by mappings, on the rule names, that define a set of rules intended for addition or deletion from the integrated framework $S P$ (the "gluing" of $S P_{1}$ and $S P_{2}$ ).

Definition 6 (meta policy). A meta policy $M P=\left(\text { strategy }_{i}\right)_{i \in I}$ for $S P$ is an I-indexed set of partial mappings strategy $y_{i}: D_{i} \rightarrow \mid$ Rules $\mid$ from a set $D_{i} \subseteq P_{1} \cup P_{2}$ to the set of rules $|\mathbf{R u l e s}|$.

A mapping strategy $: D \rightarrow \mid$ Rules $\mid$ of a meta policy changes the framework $S P$ with respect to the rules by deleting all rules from $S P$ that occur in $D$ and by adding the rules in the image strategy $(D)$. After a framework is modified for all mappings in a meta policy $M P$, it is called closed under $M P$.

Definition 7 (closure under meta policy). Let $S P$ be the integrated framework for $S P_{1}$ and $S P_{2}$ and $M P=\left(\text { strategy }_{i}: D_{i} \rightarrow \mid \text { Rules } \mid\right)_{i \in I}$ a meta policy for $S P$. The framework $S P^{M P}=\left(T G^{i n t},\left(P^{M P}, r_{P^{M P}}\right), P_{o s}{ }^{i n t}, N e g^{i n t}\right)$ closed under $M P$ consists of the set of rules names

$$
P^{M P}=P^{i n t} \backslash \bigcup_{i \in I} D_{i} \cup \bigcup_{i \in I} \operatorname{dom}\left(\text { strategy }_{i}\right)
$$

The mapping $r_{P^{M P}}: P^{M P} \rightarrow|\mathbf{R u l e s}|$ is defined for $p \in P^{\text {int }}$ as $r_{P^{M P}}=r_{P^{\text {int }}}(p)$ and for $p \notin P^{\text {int }}$ as $r_{P^{M P}}(p)=$ strategy $_{i}(p)$, where $p \in \operatorname{dom}\left(\right.$ strategy $\left._{i}\right)$.

For the following examples we introduce the weakrule $p_{2}\left(p_{1}\right)$ of a rule $p_{2}$ w.r.t. a rule $p_{1}$. The weakrule is derived from rule $p_{2}$ by adding a negative application condition $W A P\left(p_{2}, p_{1}\right)$, that allows the application of the rule $p_{2}$ only if the rule $p_{1}$ is not applicable. The application condition $W A P\left(p_{2}, p_{1}\right)$ is constructed by extending the left-hand side $L_{2}$ of $p_{2}$ to $L_{1} \cup L_{2}$.

Definition 8 (weak rule). Given two rules $p_{1}: L_{1} \stackrel{r_{1}}{R_{1}}$ and $p_{2}: L_{2} \stackrel{r_{2}}{\underline{n}}$ $R_{2}$, the weak condition for $p_{2}$ w.r.t. $p_{1}$, denoted by $W A P\left(p_{2}, p_{1}\right)$, is the pair $\left(L_{2}, L_{1} \cup L_{2}\right)$. The weakrule $p_{2}\left(p_{1}\right)$ of $p_{2}$ w.r.t. $p_{1}$ is the rule $p_{2}$ extended by the negative application condition $W A P\left(p_{2}, p_{1}\right)$.

Example 7 (weak rule). Figure 10 shows the example of the ACL rule create object and the LBAC rule new object (note that the rule LBAC rule is typed over the integrated type graph, with a $P x$ node instead of a $S x$ node). The weak rule for create object w.r.t. new object has a NAC that forbids the application to a process with a security level. Therefore, the weak rule for create object is only applicable to processes coming from the ACL model and without a counterpart in the LBAC model. The weak rule for new object w.r.t. create object has a NAC that forbids the application if a user is connected to the process. Since each user is connected to a process, the rule is not applicable. 


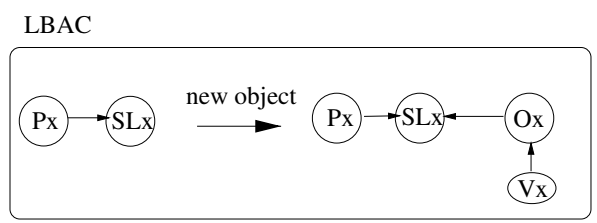

weak "new object" w.r.t. "create object"

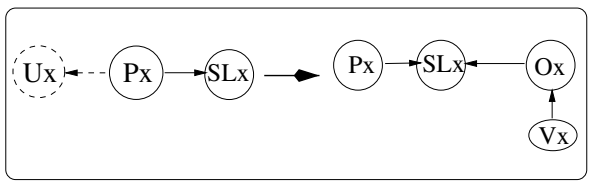

ACL

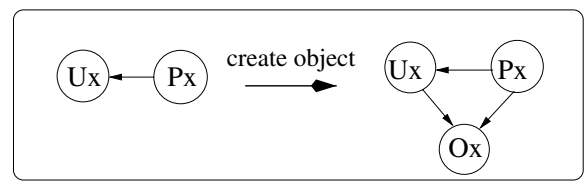

weak "create object" w.r.t. "new object"



Fig. 10. The weak rule for new object and create object.

The weak rule ensures that the less important (minor) rule of $S P_{2}$ is applied only if the more important (major) rule of $S P_{1}$ is not applicable.

Lemma 1. The weak rule $p_{2}\left(p_{1}\right)$ is applicable at a match $m: L_{2} \rightarrow G$ if and only if the morphism $m$ cannot be extended to a match for $p_{1}$.

We introduce three possible meta policies, called radical, weakRadical and static. The meta policies radical and weakRadical choose a major $S P_{1}$ and a minor policy $S_{2}$. The meta policy radical solves the problem of conflicting rules globally by selecting the rules of $S P_{1}$ and deleting the rules of $S P_{2}$. It consists of the mapping killMinor : $P_{2} \rightarrow \mid$ Rules $\mid$ defined as identity for the rules of $S P_{2}$ not in a conflict with a rule in $S P_{1}$.

$$
\operatorname{killMinor}\left(p_{2}\right)= \begin{cases}\text { undefined } & \text {, if there is }\left(p_{1}, p_{2}\right) \in C R(S P) \\ p_{2} & \text {, otherwise }\end{cases}
$$

The effect of the meta policy radical is that $S P_{1}$ will "survive" during the subsequent evolution of the system, whereas the framework $S P_{2}$ "dies". The meta policy weakRadical keeps the conflicting rule of $S P_{2}$, extended by all NACs of the weak rule w.r.t. conflicting rules, and consists of the mapping weakMinor $: P_{2} \rightarrow \mid$ Rules $\mid$ defined by

$$
\text { weakMinor }\left(p_{2}\right)=p_{2} \text { with weak condition } W A P\left(p_{1}, p_{2}\right) \forall\left(p_{1}, p_{2}\right) \in C R(S P)
$$

To non-conflict points, the weakened rule $p_{2}$ is still applicable so that the part of the system graph where the rule $p_{2}$ is applicable is only reduced.

Whereas the meta policies radical and weakRadical favor the major framwork $S P_{1}$, the choice in the meta policy static depends on the conflicting rule pair. The meta policy static consists of the two mappings weakMinor $: D_{2} \rightarrow$ $\mid$ Rules $\mid$ as above with a domain $D_{2} \subseteq P_{2}$ and weakMajor : $D_{1} \rightarrow \mid$ Rules $\mid$ with $D_{1} \subseteq P_{1}$ similar to the mapping weakMinor, defined by

$$
\text { weakMajor }\left(p_{1}\right)=p_{1} \text { with weak condition } W A P\left(p_{2}, p_{1}\right) \forall\left(p_{1}, p_{2}\right) \in C R(S P)
$$

The meta policy weakRadical is a special case of the meta policy static, where $D_{2}=P_{2}$ and $D_{1}=\emptyset$. 
Proposition 5 (conflictfreenes). The closure of the framework SP under the metapolicies radical or weakRadical is conflictfree. If $D_{1} \cap D_{2}=\emptyset$ and $D_{1} \cup$ $D_{2}=\left\{p, p^{\prime} \mid\left(p, p^{\prime}\right) \in C R(S P)\right\}$, then the closure under static is conflictfree.

\section{Concluding Remarks}

We have presented a formalism to specify AC policies. States are represented by graphs and their evolution by graph transformations. A policy is formalized by four components: a type graph, positive and negative constraints (a declarative way of describing what is wanted and what is forbidden) and a set of rules (an operational way of describing what can be constructed). The change over time of a policy can described in terms of sequences of framework morphisms, corresponding to step-by-step addition/deletion of rules and constraints.

We have also discussed the effect of integrating two policies using a pushout in the category of policy frameworks and framework morphisms. The problem of dealing with inconsistencies caused by conflicts between a rule of one policy and a constraint of the other policy has been addressed in part elsewhere KMPP00b, KMPP00a, where it is also shown the adequacy of this framework to represent different Access Control policies.

Besides the new results in Sections 3 and 4, we have introduced the notion of metapolicy and shown that three natural ones transform a policy resulting from an integration into a conflict-free policy. The choice of the appropriate meta policy may depend on the specific application domain of the particular AC model. Among the problems still under investigation are the transition from a system using one policy to a system using another policy.

\section{References}

[BdVS00] P. Bonatti, S. De Capitani di Vimercati, and P. Samarati. A modular approach to composing access control policies. In Proc. of the 7th ACM Conference on Computer and Communication Security. ACM, November 2000 .

[CELP96] A. Corradini, H. Ehrig, M. Löwe, and J. Padberg. The category of typed graph grammars and their adjunction with categories of derivations. In 5th Int. Workshop on Graph Grammars and their Application to Computer Science, number 1073 in LNCS, pages 56-74. Springer, 1996.

$\left[\mathrm{EHK}^{+} 97\right]$ H. Ehrig, R. Heckel, M. Korff, M. Löwe, L. Ribeiro, A. Wagner, and A. Corradini. Handbook of Graph Grammars and Computing by Graph Transformations. Vol. I: Foundations, chapter Algebraic Approaches to Graph Transformation Part II: Single Pushout Approach and Comparison with Double Pushout Approach. In Rozenberg [Roz97], 1997.

[GRPPS98] M. Große-Rhode, F. Parisi-Presicce, and M. Simeoni. Spatial and Temporal Refinement of Typed Graph Transformation Systems. In Proc. of MFCS'98, number 1450 in LNCS, pages 553-561. Springer, 1998.

[HW95] R. Heckel and A. Wagner. Ensuring consistency of conditional graph grammars - a constructive approach. In Proc. SEGRAGRA'95 Graph Rewriting and Computation, number 2. Electronic Notes of TCS, 1995. http://www.elsevier.nl/locate/entcs/volume2.html. 
[KMPP00a] M. Koch, L. V. Mancini, and F. Parisi-Presicce. On the specification and evolution of access control policies. Technical Report SI-2000/05, Dip.Scienze dell'Informazione, Uni. Roma La Sapienza, May 2000.

[KMPP00b] M. Koch, L.V. Mancini, and F. Parisi-Presicce. A Formal Model for Role-Based Access Control using Graph Transformation. In F.Cuppens, Y.Deswarte, D.Gollmann, and M.Waidner, editors, Proc. of the 6th European Symposium on Research in Computer Security (ESORICS 2000), number 1895 in LNCS, pages 122-139. Springer, 2000.

[PPEM87] F. Parisi-Presicce, H. Ehrig, and U. Montanari. Graph Rewriting with unification and composition. In H. Ehrig, M. Nagl, G. Rozenberg, and A. Rosenfeld, editors, Int. Workshop on Graph Grammars and their Application to Computer Science, number 291 in LNCS, pages 496-524. Springer, 1987.

[Roz97] G. Rozenberg, editor. Handbook of Graph Grammars and Computing by Graph Transformations. Vol. I: Foundations. World Scientific, 1997.

[San93] R. S. Sandhu. Lattice-based access control models. IEEE Computer, 26(11):9-19, 1993.

[San98] R. S. Sandhu. Role-Based Access Control. In Advances in Computers, volume 46. Academic Press, 1998.

[SS94] R.S. Sandhu and P. Samarati. Access Control: Principles and Practice. IEEE Communication Magazine, pages 40-48, 1994. 